\title{
ASSESSMENT AND INVESTIGATION OF STROKE AND TRANSIENT ISCHAEMIC ATTACK
}

$\Delta$ ny discussion of the diagnosis and investigation of stroke and transient ischaemic attack (TIA) must keep in mind the convincing evidence of the benefits of organised stroke care to patients of all ages, ${ }^{1}$ the availability of evidence based guidelines for management of patients with stroke, ${ }^{2}{ }^{3}$ and the evidence that the management of substantial numbers of patients does not reach these standards. ${ }^{4}$ As a consequence of the above, purchasers of health care are increasingly demanding that there is a coherent strategy for stroke care in their area, and current patterns of referral will undoubtedly change. As an individual practitioner you need to be aware how and where your skills will contribute to the overall multidisciplinary team approach to stroke care. Will you be assessing patients hyperacutely in the emergency room (minutes to hours after onset), subacutely as inpatient referrals or rapid access outpatient clinic (hours to days after onset), or will it only be the occasional, unusual patient referred for a tertiary opinion? You will also need to be conversant with the skills of other multidisciplinary team members, something that lies outside the traditional repertoire of many clinical neurologists.

Despite both technological and therapeutic advances, the clinical history and examination remain central to making an accurate, prompt, complete diagnosis. Additionally, the manner of the personal contact with a doctor at a time when patients are feeling particularly vulnerable should not be underestimated - there are few things that people fear more than serious disability from a stroke. However, you must remember that the priorities of patients and carers may differ from those of professionals, and while they clearly value being treated by knowledgeable staff, this knowledge has to be communicated in a consistent and appropriate way that allows them to participate in shared decision making. ${ }^{1}$

\section{DIAGNOSIS}

To make a complete diagnostic evaluation in a patient presenting with a possible cerebrovascular event, all the questions in the box below need to be answered. The information to do this will not all be available immediately and therefore it can be useful to go through these questions each time the patient is re-assessed.

\section{Questions to be answered in patients with suspected cerebrovascular disease}

- Is it a vascular event?

- Where in the brain has the vascular event occurred (parenchymal and vascular topography)?

- What type of vascular event is it (pathology)?

- What has caused the vascular event (mechanism)?

- What are the consequences of the vascular event (impairments, disabilities, and handicap)?

- What other medical problems co-exist?

Correspondence to: Dr John Bamford, Department of Neurology, St James's University Hospital, Beckett Street, Leeds LS9 7TF, UK

j.m.bamford@leeds.ac.uk

\section{Is it time to change the definitions of stroke and TIA?}

The changing therapeutic environment means that for the first time in over a quarter of a century we need to look critically at the "standard" definition of stroke. ${ }^{56}$ The inclusion of subarachnoid haemorrhage now seems inappropriate since the diagnostic, management, and research implications are quite distinct from those of other forms of stroke. However, perhaps the most problematic area concerns the time based definitions of stroke and TIA (that is, symptoms of more or less than 24 hours' duration) given that patients are increasingly being seen-and in some cases treated with potentially dangerous drugs-within a few hours of the onset of symptoms. Of course, in earlier schemes of classification attacks of "transient cerebral ischemia without infarction" were considered to last " 10 seconds, 10 minutes or even an hour", a definition 
Table 1 The differential diagnosis of focal neurological symptoms. Adapted from Warlow et $\mathrm{al}^{8}$

\begin{tabular}{|c|c|c|c|}
\hline & TIA & $\begin{array}{l}\text { Brain } \\
\text { attack }\end{array}$ & Stroke \\
\hline Migraine & +++ & ++ & + \\
\hline Epilepsy: partial seizure/Todd's paresis & +++ & ++ & + \\
\hline Transient global amnesia & +++ & ++ & - \\
\hline \multicolumn{4}{|l|}{ Structural intracranial lesions } \\
\hline Subdural haematoma & ++ & ++ & ++ \\
\hline Tumour & + & ++ & ++ \\
\hline Arteriovenous malformation/aneurysm & ++ & ++ & + \\
\hline \multicolumn{4}{|l|}{ Metabolic/toxic disorders } \\
\hline $\begin{array}{l}\text { Hypoglycaemia and other acute metabolic } \\
\text { disorders }\end{array}$ & +++ & ++ & + \\
\hline Hypertensive and other encephalopathies & + & ++ & ++ \\
\hline CNS infections: encephalitis/brain abscess & + & ++ & ++ \\
\hline \multicolumn{4}{|l|}{ Labyrinthine disorders } \\
\hline Vestibular neuronitis & + & ++ & ++ \\
\hline $\begin{array}{l}\text { Benign paroxysmal positional } \\
\text { vertigo/Ménière's disease }\end{array}$ & ++ & - & - \\
\hline \multicolumn{4}{|l|}{ Psychological disorders } \\
\hline Hyperventilation/panic attacks & +++ & - & - \\
\hline Somatisation/conversion disorder & +++ & ++ & ++ \\
\hline Head injury & + & + & + \\
\hline Multiple sclerosis & + & ++ & ++ \\
\hline \multicolumn{4}{|l|}{ Neuromuscular disorders } \\
\hline Mononeuropathy/radiculopathy & ++ & ++ & ++ \\
\hline Myasthenia gravis & ++ & - & ++ \\
\hline
\end{tabular}

+++ common or frequent; ++ encountered regularly in a busy practice or important, treatable condition; + infrequent; - rare.

which actually includes the majority of patients who ultimately are classified as having a TIA by current definitions. $^{7}$

Looked at from the perspective of physicians assessing patients within 24 hours from onset of symptoms, there are basically two groups of patients: those whose symptoms have resolved and for whom the term TIA is entirely appropriate; and those who still have symptoms with or without relevant physical signs. For this latter group there may be value in using a new term such as "brain attack", not least because it would flag up issues such as the scope of the differential diagnosis which differs from patients with completed stroke-that is, a patient with symptoms which have lasted more than 24 hours (table 1). ${ }^{8}$ Despite the advances in magnetic resonance imaging, I do not believe we are yet at the stage where the clinical basis of the definition of a cerebrovascular event should be discarded.

At present there are few data concerning in-hospital diagnostic accuracy in the "brain attack" group. In 1999 Allder and colleagues reported a $8.6 \%$ rate of clinical misdiagnosis in a study of 70 patients who were thought to have carotid distribution ischaemia and were imaged at a mean of 11.4 hours post-onset, ${ }^{9}$ which is not substantially different from previous reports in patients with completed stroke seen at a mean of four days after the ictus. ${ }^{10}$ Of some interest to those involved in triaging patients for acute treatments, data have been reported for the pre-hospital diagnosis of hyperacute cerebrovascular disease by paramedical personnel using a variety of protocols with positive predictive values of about $70-80 \% .^{11-13}$

\section{Can you localise the lesion clinically?}

Traditionally, clinical symptoms and signs are used to localise the lesion in the brain. Lesions at certain sites are then grouped together according to their presumed common vascular supply, and, sometimes, inferences are made about the underlying mechanism of the stroke. The distinction is usually made between anterior (carotid) and posterior (vertebrobasilar) distribution events, and this is used to make clinically important decisions such as whether a patient should undergo carotid duplex examination or whether a high grade carotid stenosis should be viewed as symptomatic or asymptomatic. Because of the qualitative differences between lacunar and cortical strokes in terms of underlying vascular disease and prognosis, some schemes of classification identify such groups separately. ${ }^{14}{ }^{15}$ Even at this rather basic level of subclassification the overall inter-rater reliability is only moderate. ${ }^{16}{ }^{17}$ Although in common with other areas of medicine relatively little is known about the reliability of individual aspects of the clinical examination, ${ }^{18}$ what studies there are suggest that even experienced clinicians have problems ${ }^{16}{ }^{19}$; however, there is evidence that, for lacunar strokes at least, the reliability is greater among clinicians with a specific interest in cerebrovascular disease. ${ }^{20}$

The situation is further complicated in the patient with a "brain attack" because the clinical symptoms and signs often change. For example, in a study of 152 patients with supratentorial ischaemic stroke who were seen within five hours of onset of symptoms, $39(26 \%)$ had some neurological deterioration during the subsequent four days. ${ }^{21}$ The same authors showed that for patients who presented with either a pure motor or sensorimotor stroke-that is, lacunar syndromes-within 12 hours of onset (mean 6.1 hours) the positive predictive value for a small, deep infarct (that is, compatible with occlusion of a single deep perforating artery) was only $56 \%$. Furthermore, they also noted that there was another group of patients who presented acutely with non-lacunar syndromes who had small, deep infarcts (equivalent to lacunar infarcts) on a second computed tomographic (CT) scan two weeks later. Of these 47 patients, 23 (49\%) improved over the following days with resolution of their "cortical" symptoms and signsthat is, if they had been examined a few days after the onset and there had been no clear record or history of the clinical pattern at the time of maximum deficit, then they would have been classified (correctly) as lacunar infarcts. ${ }^{22}$

While there are still many reasons why it would not be appropriate to use conventional CT or even magnetic resonance imaging alone as the method of anatomical localisation in acute stroke (not least being the difficulty of identifying the relevant lesion), there is some evidence to suggest that if appropriate abnormalities are imaged then they should be used to modify the syndromic localisation. ${ }^{23} 24$

\section{Investigation}

How far one investigates an individual patient for the cause of their stroke or TIA depends on how the information would inform management decisions, hopefully evidence based ones! This will be influenced by a range of factors, not least their current and pre-morbid levels of disability. It is always worth keeping in mind that in population studies about $95 \%$ of ischaemic events are caused by either atherothromboembolism, cardiogenic embolism or intracranial small vessel disease, and that absolute proof of the stroke mechanism in an individual patient will rarely be available as many patients have multiple potential mechanisms. ${ }^{25}$ For example, while stroke in the presence of atrial fibrillation is usually taken as a strong pointer to cardiogenic embolism, about $10 \%$ of sufferers will have had a cerebral haemorrhage and up to $20 \%$ will have significant carotid stenosis, aortic arch atheroma or intracranial small vessel disease. ${ }^{26-29}$ Therefore, unless there are very strong clinical pointers to a rare mechanism of stroke, the initial investigations should be confined to those that could positively support such mechanisms rather than simply exclude rarities. As the clinical and investigational evidence 
Table 2 The probable yield of routine investigations. Adapted from Warlow et $a l^{8}$

\begin{tabular}{lc}
\hline Investigation & $\begin{array}{c}\text { Estimated } \\
\text { yield (\%) }\end{array}$ \\
\hline Full blood count & 1 \\
ESR/plasma viscosity & 2 \\
Urea and electrolytes & 3 \\
Plasma glucose & 5 \\
Plasma cholesterol & 45 \\
Urinalysis & 5 \\
ECG (12 lead) & 17 \\
Unenhanced CT brain scan & 20
\end{tabular}

$\overline{\text { ESR, erythrocyte sedimentation rate; ECG, electrocardiogram; CT computed }}$ tomography.

accumulates, clinicians should always be asking themselves whether the point has been reached where there is reasonable support for a particular mechanism, in which case further investigation is not justified. Indeed, it is not acceptable to subject patients or the healthcare system to the risks and costs of undirected cerebrovascular philately.

\section{Doing the basics properly}

Certain investigations should be performed almost immediately on (virtually) all patients (table 2). Although the estimated yield (that is, the proportion of patients in whom a positive test result may lead to a useful change in management) is often quite low, the tests are by and large inexpensive and the consequences of missing a treatable diagnosis such as giant cell arteritis or diabetes may be serious. Table 2 does not include either syphilis serology or routine chest radiography. Kelley and colleagues reported that even though screening tests for syphilis may be positive the detection of unexpected meningovascular syphilis was very uncommon. ${ }^{30} \mathrm{~A}$ similar pattern was found in the Oxfordshire community stroke project. It may be, therefore, that this investigation should not be performed routinely but should be targeted at high risk populations. Sagar and colleagues concluded that admission chest radiography was not indicated-films were frequently of rather poor quality because of difficulties with patient positioning and inspiration, and it would seem better to confine requests to those patients with clear clinical indications. ${ }^{31}$

\section{Ultrasound investigations}

Ultrasound investigations should be used more selectively, to a large extent guided by the results of the clinical examination and the results of cross sectional imaging. Carotid duplex is an appropriate examination in patients with clinico-radiological evidence of carotid territory ischaemia who would accept, and be accepted for, carotid endarterectomy or angioplasty if an appropriate lesion was detected. The place of vertebral duplex is less clear since the vertebral artery origins where atheroma may form are not easily visualised, there is also the problem of substantial inter-individual anatomical variability which can hinder the interpretation of any changes that are detected. The (rare) subclavian steal syndrome can be suspected clinically by measuring the blood pressure in both arms; however, it is worth remembering that asymptomatic reversal of flow in one vertebral artery is a relatively common ultrasound finding. ${ }^{32}$

The role of transcranial Doppler in routine rather than research practice is still evolving. It has the potential to provide a non-invasive, repeatable, and relatively inexpensive examination at the bedside which can give information about intracranial stenoses or occlusions (which may become an important factor in patients being considered for thrombolysis), ongoing asymptomatic embolism (which may allow stratification of subsequent stroke risk), the possibility of paradoxical embolisation, and the cerebrovascular reserve. ${ }^{33}$ The problems include the $5-10 \%$ of patients who have no acoustic window, the low spatial resolution, the variability of diagnostic criteria, and the dependence on the skill of the operator.

The role of transthoracic echocardiography in the majority of patients with ischaemic stroke is to guide the management of cardiac conditions that have been identified by the clinical examination, ECG, and chest radiograph. This would include suspected valve lesions and symptomatic left ventricular dysfunction. Echocardiography should be performed in cases of possible endocarditis and also if there has been evidence of embolism to other parts of the body. Many cardiologists would want echocardiography before making a decision about attempted cardioversion in patients in atrial fibrillation. ${ }^{34}$ Echocardiography performed in the absence of other pointers to cardiac disease is not justified because of the very low yield of information that would change clinical management. One exception is the younger patient with no other explanation for their ischaemic stroke, when detection of lesions such as a patent foramen ovale or atrial septal aneurysm, or both, may be relevant. However, in this situation the clinician must be willing to subject the patient to transoesophageal echocardiography with contrast agents, a procedure that carries a small risk..$^{35}$

\section{Summary}

- There are now several evidence based guidelines for the management of patients with stroke

- The concept of a "brain attack" will need to be developed for patients with persisting symptoms who are seen $<24$ hours from onset

- Overall about $95 \%$ of ischaemic events are caused by either atherothromboembolism, cardiogenic embolism or intracranial small vessel disease

- Absolute proof of the stroke mechanism in an individual patient will rarely be available as many patients have multiple potential mechanisms

- Carotid duplex is an appropriate examination in patients with clinico-radiological evidence of carotid territory ischaemia who would accept, and be accepted for, carotid endarterectomy or angioplasty if an appropriate lesion was detected

- Transcranial Doppler has the potential to provide a noninvasive, repeatable, and relatively inexpensive bedside examination which can give information about intracranial stenoses or occlusions, vasospasm, asymptomatic embolism, paradoxical embolisation, and the cerebrovascular reserve

- Routine echocardiography in the absence of other pointers to cardiac disease is not justified because of the very low yield of information that would change clinical management

\section{References}

1 Stroke Unit Trialists' Collaboration. Organised inpatient (stroke unit) care for stroke (Cochrane Review). In: The Cochrane Library, Issue 3 , 2000. Oxford: Update Software.

2 The Intercollegiate Working Party for Stroke. National clinical guidelines for stroke. London: Royal College of Physicians, 2000.

3 Scottish Intercollegiate Guidelines Network. Management of patients with stroke. 1. Assessment, investigation, immediate management and secondary prevention. Edinburgh: SIGN. Publication No 13, 1997.

4 Rudd AG, Irwin P, Rutledge Z, et al. The national sentinel audit of stroke: a tool for raising standards of care. $J R$ Coll Physicians Lond 1999;33:460-4. 
5 Hatano S. Experience from a multicentre stroke register: a preliminary report. Bull World Health Organ 1976;54:541-53.

6 Advisory Council for the National Institute of Neurological Diseases and Blindness. A classification and outline of cerebrovascular diseases. Stroke 1975;6:564-616.

7 Advisory Council for the National Institute of Neurological Diseases and Blindness. A classification and outline of cerebrovascular diseases. Neurology 1958;8:395-434.

8 Warlow CP, Dennis MS, van Gijn J, et al. Stroke: a practical guide to management, 2nd ed. Oxford: Blackwell Science, 2000.

9 Allder SJ, Moody AR, Martel AL, et al. Limitations of clinical diagnosis in acute stroke. Lancet 1999;354:1523.

10 Sandercock P, Molyneux A, Warlow C. Value of computed tomography in patients with stroke: Oxfordshire community stroke project. BMJ 1985;290:193-7.

11 Kothari R, Barsan W, Brott T, et al. Frequency and accuracy of prehospital diagnosis of acute stroke. Stroke 1995;26:937-41.

12 Kidwell CS, Starkman S, Eckstein M, et al. Identifying stroke in the field. Prospective validation of the Los Angeles prehospital stroke screen (LAPSS). Stroke 2000;31:71-6.

13 Harbison J, Massey A, Barnett L, et al. Rapid ambulance protocol for acute stroke. Lancet 1999;353:1935.

14 Bamford J, Sandercock P, Jones L, et al. The natural history of lacuna infarction: the Oxfordshire community stroke project. Stroke 1987; 18:545-51.

15 Bamford J, Sandercock P, Dennis M, et al. Classification and natura history of clinically identifiable subtypes of cerebral infarction. Lancet 1991;337:1521-6.

16 Lindley RI, Warlow CP, Wardlaw JM, et al. Interobserver reliability of a clinical classification of acute cerebral infarction. Stroke 1993;24:1801-4.

17 Lindgren A, Norrving B, Rudling O, et al. Comparison of clinical and neuroradiological findings in first-ever stroke: a population-based study. Stroke 1994;25:1371-7.

18 McAlister FA, Straus SE, Sackett DL on behalf of the CARE-COAD1 Group. Why we need large, simple studies of the clinical examination: the problem and a proposed solution. Lancet 1999;354:1721-4.

19 Shinar D, Gross CR, Mohr JP, et al. Interobserver variability in the assessment of neurologic history and examination in the stroke data bank. Arch Neurol 1985;42:557-65.

20 Boiten J, Lodder J. Lacunar infarcts: pathogenesis and validity of the clinical syndromes. Stroke 1991;22:1374-8.
21 Toni D, Fiorelli $M$, Gentile $M$, et al. Progressing neurological deficit secondary to acute ischemic stroke. A study on predictability, pathogenesis, and prognosis. Arch Neurol 1995;52:670-5.

22 Toni D, Del Duca R, Fiorelli M, et al. Pure motor hemiparesis and sensorimotor stroke. Accuracy of very early clinical diagnosis of lacunar strokes. Stroke 1994:25:92-6.

23 Mead GE, Lewis SC, Wardlaw JM, et al. Should computed tomography appearance of lacunar stroke influence patient management? J Neurol Neurosurg Psychiatry 1999;67:682-4.

24 Mead GE, Lewis SC, Wardlaw JM, et al. How well does the Oxfordshire community stroke project classification predict the site and size of the infarct on brain imaging? J Neurol Neurosurg Psychiatry 2000;68:558-62.

25 Caplan LR. Lice, fleas, and strokes. Arch Neurol 2000;57:1113-14.

26 Lodder J, Bamford JM, Sandercock PAG, et al. Are hypertension or cardiac embolism likely causes of lacunar infarction. Stroke 1990;21:375-81.

27 Bogousslavsky J, van Melle G, Regli F, et al. Pathogenesis of anterior circulation stroke in patients with nonvalvular atrial fibrillation: the Lausanne stroke registry. Neurology 1990;40:1046-50.

28 Lindgren A, Roijer A, Norrving B, et al. Carotid artery and heart disease in subtypes of cerebral infarction. Stroke 1994;25:2356-62.

29 Mead GE, Murray H, Farrell A, et al. Pilot study of carotid surgery for acute stroke. Br J Surg 1997;84:990-2.

30 Kelley RE, Bell L, Kelley SE, et al. Syphilis detection in cerebrovascular disease. Stroke 1989;20:230-4.

31 Sagar G, Riley P, Vohrah A. Is admission chest radiography of any clinical value in acute stroke patients? Clinical Radiology 1996:51:499-502.

32 Hennerici M, Klemm C, Rautenberg W. The subclavian steal phenomenon: a common vascular disorder with rare neurologic deficits. Neurology 1988;38:669-73.

33 Markus HS. Transcranial Doppler ultrasound. J Neurol Neurosurg Psychiatry 1999;67:135-7.

34 Cheitlin MD, Alpert JS, Armstrong WF, et al. ACC/AHA guidelines for the clinical application of echocardiography: executive summary. A report of the American College of Cardiology/American Heart Association task force on practice guidelines. J Am Coll Cardiol 1997;29:862-79.

35 Daniel WG, Mugge A. Transesophageal echocardiography. N Engl J Med 1995;332:1268-79.

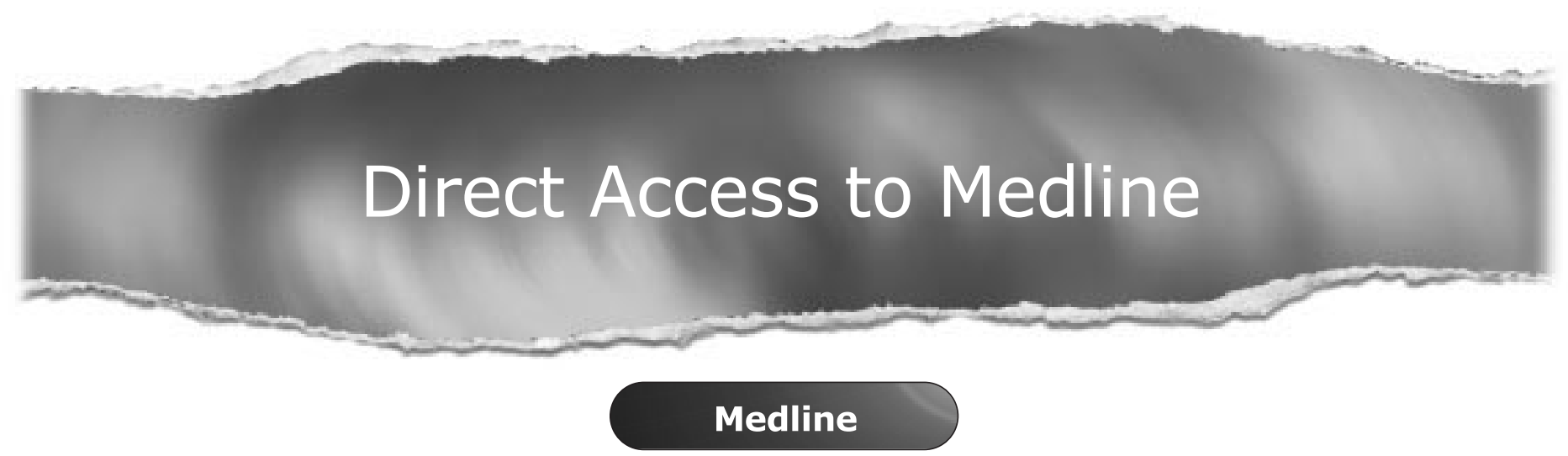

Link to Medline from the homepage and get straight into the National Library of Medicine's premier bibliographic database. Medline allows you to search across 9 million records of bibliographic citations and author abstracts from approximately 3,900 current biomedical journals.

\section{www.jnnp.com}

